\title{
Probleme der Historiographie der Wissenschaften in islamischen Gesellschaften vor 1700
}

Sonja Brentjes

Glen van Brummelen 2009: The Mathematics of the Heavens and the Earth. The Early History of Trigonometry. Princeton: Princeton University Press. 352 S., 42,00 \$, ISBN-13: 978-0-691-12973-0.

Amir Ljubović 2008: The Works in Logic by Bosniac Authors in Arabic. Leiden: Brill. 254 S., 96,00 €, ISBN-13: 978-90-04-16856-5.

Robert G. Morrison 2007: Islam and Science, The intellectual career of Niz̄ām al-Dīn al-Nīsābūrī. New York/London: Routledge. 312 S., 22,50 f, ISBN-13: 978-0415663991.

Ulrich Rudolph 2008: Islamische Philosophie. Von den Anfängen bis zur Gegenwart. München: C. H. Beck, (2. Aufl.). 123 S., 8,95 €, ISBN-13: 978-3-406-50852-3.

George Saliba 2007: Islamic Science and the Making of the European Renaissance. Cambridge, Massachusetts: MIT Press. 327 S., ISBN-13: 978-026251615-0.

Petra G. Schmidl 2007: Volkstümliche Astronomie im islamischen Mittelalter. Zur Bestimmung der Gebetszeiten und der Qibla bei al-Aṣbaḥī, Ibn Raḥīq und al-Fārisī, Leiden: Brill, geb., 904 S., 2 Bde. 325,00 €, ISBN-13: 978-90-04-15390-5.

Uwe Vagelpohl 2008: Aristotle's Rhetoric in the East. The Syriac and Arabic Translation and Commentary Tradition. Leiden: Brill. 347 S., 117,99 €, ISBN-13: 978-900416681-3. 
Die Geschichte der Wissenschaften in islamischen Gesellschaften als akademisches Fach, Teil der Wissenschaftsgeschichte ebenso wie der verschiedenen historischen Disziplinen, die sich mit islamischen Gesellschaften beschäftigen, wie zum Beispiel Islamwissenschaften, Arabistik, Iranistik, Ottomanistik oder anderen Formen von Regionalgeschichte, ist eine widersprüchliche, zumeist instabile Geschichte, die auf das Engste mit den Werturteilen über islamische Gesellschaften, die Rolle der Wissenschaften in ihnen und deren Bedeutung für die Wissenschaften in nicht-islamischen Gesellschaften in Europa verbunden war und ist. Nach Jahrzehnten eines produktiven und viel beachteten Aufschwungs im methodologischen Rahmen internalistischer Analysen in der zweiten Hälfte des 20. Jahrhunderts befindet sie sich seit mehreren Jahren in einer Krise, die ich für schwerwiegend und bedrohlich für die Existenz des Faches halte. Die anhaltende Konzentration auf internalistische Fragestellungen und Darstellungsweisen hat im Zusammenspiel mit den tiefgreifenden methodologischen Änderungen in anderen Bereichen der Wissenschaftsgeschichte, mit grundsätzlichen Verschiebungen von Urteilen über die Geschichte der Philosophie in islamischen Gesellschaften und mit politischen und ökonomischen Entscheidungen vor allem in Europa und Nordamerika zu einem schmerzhaften Rückgang akademischer Positionen für dieses Fach und einer kommunikativen Isolierung von seinen unmittelbaren Nachbarfächern geführt.

Die politische Entwicklung des letzten Jahrzehnts hat das ihrige dazu beigetragen, dass die Geschichte der Wissenschaften in islamischen Gesellschaften heute in bisher unbekanntem Ausmaß zu einer Domäne ideologischer Auseinandersetzungen geworden ist, die in wichtigen öffentlichen Räumen stattfinden und $\mathrm{zu}$ einer relativen Fülle von Publikationen (Bücher, Videos, Ausstellungen) geführt haben. Viele dieser Publikationen propagieren eine Sicht auf diese Geschichte, die ebenso problematisch ist wie der vielbeklagte und nach wie vor nicht überwundene Eurozentrismus. Seit Jahrzehnten wurden im Schulunterricht in säkularen wie religiös verfassten Ländern der sogenannten islamischen Welt Auffassungen gelehrt, die einen prinzipiell rationalen und wissensfreundlichen Charakter islamischer Religion(en), die Schaffung der modernen Wissenschaften durch muslimische Gelehrte und den Niedergang derselben entweder durch Obskurantismus, Nomadeneinfälle und Pest oder durch Kolonialismus und Okkupation zum Gegenstand hatten. Die Veröffentlichungen der letzten Dekade haben diese oft sehr einseitigen, ahistorischen Darstellungen zu einer Anklage des ,Westens' überhöht, der diese vorgeblich ,modernen' Wissenschaften ,übernommen' (manche sagen auch ,gestohlen') hat und dies seit ungefähr 1000 Jahren verschweigt. Das Fehlen verlässlicher, methodologisch anspruchsvoller, doch gleichzeitig dem allgemein gebildeten Publikum verständlicher akademischer Stimmen in diesen öffentlichen Räumen ist ebenso bedenklich wie die Zunahme ideologischer Trivialisierungen historiographischer Interpretationen in akademischen 
Publikationen. Aus diesen Gründen stehen historiographische Aspekte der ausgewählten Bücher im Mittelpunkt der folgenden Diskussion.

\section{Kontroverse Sichtweisen}

Zwei zentrale Gegenstände bisheriger Wissenschaftsgeschichtsschreibung für islamische Gesellschaften waren die Interpretation der Übersetzungen antiker und frühmittelalterlicher wissenschaftlicher Werke aus dem Griechischen, Mittelpersischen, Syrischen und Sanskrit vom 8. bis zum 10. Jahrhundert und das Problem des zumeist sehr elementaren Inhalts einer Vielzahl von Schriften, die aus der Zeit vom 13. bis zum frühen 20. Jahrhundert erhalten sind. Der erste Gegenstand ist in der Regel als ,Übersetzungsbewegung' bekannt; der zweite als ,Niedergang. Die drei ersten Autoren (Saliba, Rudolph, Vagepohl), die ich vorstelle, versuchen sich diesen Gegenständen auf neue Art und Weise zu nähern. Die Resultate könnten nicht unterschiedlicher ausgefallen sein. Während Rudolph die weitgehende Neuorientierung in der Philosophiegeschichte einem breiten Publikum vorstellt und Vagelpohl sich um die Anwendung von Methoden aus der zeitgenössischen Übersetzungstheorie bemüht, stellt Saliba eine inzwischen äußerst erfolgreiche, aber aus meiner Sicht mehr als problematische Kritik von Auffassungen früherer Autoren zu den Faktoren, die die Übersetzungsbewegung ermöglicht haben, vor. Sie leitet seinen Nachdruck verschiedener, im Kern bereits veröffentlichter Texte ein, dem eine aus anderen methodischen Gründen gleichfalls problematische Diskussion des ,Niedergangs' sowie der möglichen historischen Einflüsse arabischer Planetenmodelle auf Nicolaus Copernicus folgt.

\section{Salibas sogenannte klassische Erzählung}

George Saliba beabsichtigt mit seinem Werk, den Zusammenhang zwischen ,islamischer Wissenschaft' und europäischer Renaissance aufzuzeigen. Im ersten Kapitel verspricht er seiner Leserschaft einen Überblick zu verschiedenen Auffassungen darüber, warum und wann die wissenschaftliche Tradition entstanden ist, die von ihm als entscheidend für die Entwicklung der ,universalen Wissenschaft in vormodernen Zeiten' angesehen wird (S. 1). Anschließend will er das Versagen dieser Erklärungsmuster kritisieren, was die Grundlagen für seine ,alternative' Erzählung im zweiten Kapitel bereitstellen soll. Dieses Versprechen löst der Autor jedoch nicht ein. Vielmehr erfindet er eine Grunderzählung, die er ,klassisch` nennt und deren Ursprünge er unzutreffend in die Jahrhunderte des Mittelalters und der Renaissance datiert. Seine Beschreibung von Versatzstücken über die Übersetzungsbewegung und die Wissenschaften in islamischen Gesellschaften ergänzt Saliba anschließend um Punkte, die erst in heutigen islamischen Gesellschaften $\mathrm{zu}$ einem festen 
Bestandteil der Erzählung über die Wissenschaften geworden sind. Salibas ,klassische Erzählung' wie seine Dekonstruktion derselben machen einen tendenziösen, übertriebenen und sarkastischen Eindruck. Der von ihm erfundenen ,klassischen Erzählung unterstellt er Behauptungen, die in seiner eigenen Darstellung nicht vorkommen und auch in der älteren Literatur in dieser Form nicht anzutreffen sind.

In seinem, dieser ,klassischen Erzählung' entgegengesetzten Erklärungsangebot stützt Saliba sich auf die Behauptung, dass heutige Interpretationen vergangener Ereignisse und Prozesse von den Beschreibungen und Erklärungen ausgehen müssen, die die historischen Interpreten derselben bereits geliefert haben (S. 28). Folgerichtig sieht er seine Alternative in elementarer Form bereits in dem Werk des von ihm als ersten ,Theoretiker der Übersetzungsbewegung' definierten Autors Ibn al-Nadīm († ca. 998) (S. 28 f.). Dieser habe schon erkannt, dass die Wissenschaften in der islamischen Zivilisation durch die administrativen Bedürfnisse des Staates zur Zeit des Umayyadenkalifen 'Abd al-Malik (reg. 685-705) ins Leben gerufen worden seien (S. 52). Saliba interpretiert diesen Beginn als Ergebnis einer scharfen Konkurrenz zwischen verschiedenen Akteursgruppen in der Verwaltung. In den restlichen fünf Kapiteln seines Buches benutzt er dieses Konzept der Konkurrenz als monokausale Erklärung für die Existenz von wissenschaftlichen Aktivitäten.

Im letzten Kapitel widmet sich Saliba in erster Linie dem ,Niedergang. Hier vertritt er die Ansicht, dass dieser Prozess von zwei weitgehend voneinander unabhängigen Ansätzen postuliert und erklärt worden sei. Der erste, der seiner Meinung nach von verschiedenen Islamwissenschaftlern seit dem 19. Jahrhundert verfolgt worden ist, besagt, dass die islamische Geschichte im Wesentlichen als eine Entfaltung der Religion zu verstehen sei. Den zweiten schreibt er Politikhistorikern zu, die den Mongoleneinfall und die Zerstörung Bagdads und des abbasidischen Kalifats im Jahre 1258 als den Kern allen Übels bestimmt hätten.

Eine Geschichte der sozio-kulturellen Kontexte wissenschaftlichen Arbeitens in islamischen Gesellschaften oder historischer Analysen dieser Arbeiten durch heutige Experten oder Laien bietet Saliba jedoch nicht. Autoren, die er ablehnt, nimmt er in seiner Analyse nicht ernst genug. Er macht sie stattdessen zu Buhmännern seiner Polemik. Die anderen, die er verteidigen und rehabilitieren will, dekontextualisiert er zu stark. Damit kann er in ihnen nicht mehr als geniale Zerstörer antiker Theorien und Begründer neuer Modelle erkennen. Obwohl im Grunde sein Appell, die vorhandenen Interpretationen der Geschichte wissenschaftlicher Aktivitäten in islamischen Gesellschaften kritisch zu befragen und durch neue Ergebnisse zu verfeinern oder zu ersetzen, gerechtfertigt und sogar überfällig ist, ist sein Buch aus den oben genannten Gründen nicht zu empfehlen. Allein der enorme (populär)wissenschaftliche Erfolg, der Islamic Science and the Making of the 
European Renaissance in den drei Jahren seit seinem Erscheinen beschieden gewesen ist, rechtfertigt seine Aufnahme in diese Rezension. Dieser außerordentliche Erfolg ist ein Indiz dafür, dass Salibas Studie drei aktuelle Bedürfnisse bedient: Erstens geht es darum, wissenschaftliche Leistungen positiv in islamischen Gesellschaften zu verankern und sie nicht länger als ,Aufbewahrung Wissens fremder Kulturen darzustellen. Zweitens entspricht Salibas Buch dem Wunsch nach einer scharfen und kritischen Zurückweisung einer einseitigen, auf den ,Westen' zentrierten Geschichtsschreibung. Drittens bietet es schließlich derzeit hoch im Kurs stehende, sozio-kulturelle Erklärungen der Wissenschaften in islamischen Gesellschaften an.

\section{Neue Ansätze in der Philosophiegeschichte}

Ulrich Rudolphs kurze Einführung in die Philosophiegeschichte richtet sich an eine allgemein gebildete Leserschaft und damit an ein ganz anderes Publikum als die anderen hier besprochenen Bücher. Deshalb ist diese Studie sehr viel bescheidener und zurückhaltender angelegt. Nach einer kurzen Darlegung des gegenwärtigen Diskussionsstandes in der Philosophiegeschichte zur Übersetzungsbewegung wendet sich der Autor in zehn knappen Kapiteln verschiedenen Philosophen und ausgewählten Schwerpunkten ihrer Lehren, ihres Zusammenhangs mit antiken Systemen, islamischen Vorgängern sowie Diskussionen in den religiösen Wissenschaften zu. Al-Kindī, al-Fārābī und Abū Bakr al-Rāzī werden sowohl als Rezipienten antiken Philosophierens als auch als Partner im religiösen Diskurs ihrer Zeit vorgestellt (S. 15-36). Er verdeutlicht damit, wie weit sich die heutige Philosophiegeschichtsschreibung von älteren Auffassungen zum Verhältnis zwischen Philosophie und Religion in islamischen Gesellschaften entfernt hat. Rudolph zufolge stellt Ibn Sīnā ein neues Paradigma auf, dass das Philosophieren in den nächsten sieben bis acht Jahrhunderten angeregt und inspiriert hat. Es wurde von seinen Nachfolgern, ob es nun Philosophen an Höfen wie Ibn Sīnā selbst oder Religionsgelehrte in Madrasas (S. 42-55) waren, aufgenommen, modifiziert, abgelehnt oder in ihr Werk integriert.

In diesem Zusammenhang markiert Rudolph drei weitere neue Positionen der Philosophiegeschichte. Ibn Sīnā wird erstens nicht länger nur als der brillante Systematiker und Kompilator antiker Lehren angesehen, sondern als Begründer eines eigenständigen philosophischen Lehrsystems (S. 44-46). Zweitens verschwindet die Philosophie nicht allmählich nach seinem Ableben im Osten des Kalifats, sondern erhält dank der begrifflichen Schärfe der Thesen Ibn Sīnās, der Einbeziehung theologischer Fragestellungen sowie der Rezeption und Verbreitung seiner Schriften durch Schüler und deren Studenten vor allem im iranischen Osten, aber auch in arabischen Regionen des Abbasidenkalifats einen großen Aufschwung (S. 47-54). Drittens werden die Religionsgelehrten in den Madrasas langfristig als die eigentlichen Erben der Philosophie Ibn Sīnās bewertet (unter anderem S. 56, 80, 86). 
Diesen späteren Aneignungen, Kritiken und Neubestimmungen widmet Rudolph folgerichtig fünf Kapitel. Dort offeriert er in knappster Form zum Beispiel neue Einschätzungen zur Rolle des bedeutenden Theologen al-Ghazālī († 1111), zum ersten Illuminationsphilosophen al-Suhrawardī († 1198) und zu den veränderten Bedingungen für das Philosophieren in Madrasas ( $S$. 56-60, 77-90). Dabei befreit er al-Ghazālī von dem im 19. und 20. Jahrhundert propagierten und maßgeblichen Vorwurf der Zerstörung von Philosophie und rationaler Wissenschaft und legt nahe, dessen Lehre der sekundären Kausalitäten neu zu überdenken. Das gibt ihm die Möglichkeit, den philosophischen Gehalt von Doktrinen aus dem sufischen Milieu zu untersuchen und die überaus fruchtbare Integration philosophischer Themen und Methoden in religiöse Studien als intellektuellen Teil einer islamischen Philosophiegeschichte ernst zu nehmen.

Nach einer kurzen Darstellung des muslimischen Philosophierens in alAndalus (S. 61-76) wendet Rudolph sich den Nachfolgern von Ibn Sīnā und Suhrawardī seit dem 13. Jahrhundert sowie den neuen Positionen von Mìr Dāmād (†1630), Mullā Sadrā (†1640) und der sogenannten Schule von Isfahan in der ersten Hälfte des 17. Jahrhunderts zu (S. 77-104). Er liefert damit eine völlig neue Periodisierung der Philosophiegeschichte in islamischen Gesellschaften und unterstreicht die Bedeutung späterer islamischer Gesellschaften. Das sind Positionen, die sich vom noch immer in der Wissenschaftsgeschichte vorherrschenden Paradigma ,Goldenes Zeitalter-Niedergang deutlich unterscheiden.

Die beiden zentralen methodologischen Ansätze in Uwe Vagelpohls Buch, das auf seine Dissertation aus dem Jahre 2003 an der University of Cambridge zurückgeht, betreffen die Übersetzungstheorie und die Praxis der Kontextualisierung. Mit Ersterem führt er in „Elemente einer komparativen Übersetzungsgrammatik“ ein und mit Letzterem beabsichtigt er, ein „neues Licht auf die inneren Arbeitsweisen der Übersetzungsbewegung und ihrer Auswirkung auf die islamische Kultur [zu] werfen" (S. ix). Dementsprechend gliedert sich das Buch in vier Kapitel, die der Textanalyse sowie der Darstellung kontextueller Aspekte gewidmet sind. Einführend gibt er einen Überblick über bisherige Erklärungen der Übersetzungsbewegung, dem er eine kurze polemische Beschreibung klassischer, insbesondere philologischer Methoden des Studiums erhaltener arabischer Übersetzungen griechischer philosophischer und anderer Werke vorangestellt hat. In seiner Darstellung des älteren philologischen Ansatzes arbeitet er leider ohne Belege und greift historiographisch wie methodisch zu kurz. Seine Kritik dient vornehmlich dem Ziel, seine methodische Anleihe in der Übersetzungstheorie zu begründen. Hätte Vagelpohl in seiner Untersuchung durch die Anwendung eines breitgefächerten Methodenspektrums aus der Übersetzungstheorie gezeigt, was hinzugewonnen werden kann, wenn theoretische Positionen ernst genommen, Kontexte einbezogen und Instrumentarien ausgeschöpft werden, hätte seine Arbeit in 
der Tat eine substantielle Bereicherung für das Studium mittelalterlicher Übersetzungen werden können. So aber ist sie vor allem eine verdienstvolle philologische Analyse eines schwierigen griechischen Textes und einer vielleicht noch schwierigeren arabischen Übersetzung.

Glen van Brummelen charakterisiert das Anliegen seiner Arbeit als eine Modernisierung Anton von Braunmühls Vorlesungen zur Geschichte der Mathematik aus dem Jahre 1900/1903, dem einzigen Buch zur Geschichte der Trigonometrie als mathematischer Disziplin in einer ,westlichen' [!] Sprache (S. xi). Diesem Anliegen gerecht zu werden, dient sein Bemühen, ,to disengage from the modern context, and to try to live the past the way the past lived it" (S. xii). Van Brummelens Anmerkungen zur Mathematikgeschichtsschreibung damals und heute dokumentieren die Probleme, die vornehmlich mathematisch ausgebildete Historiker/innen der islamischen Gesellschaften mit heutigen Trends und insbesondere einer kulturellen Kontextualisierung der Geschichte der Mathematik haben. Sein starkes methodologisches Credo lautet: „One cannot genuinely practice the history of a scientific subject without also living and breathing the science itself." (S. xii) Diese beiden Leben eines Mathematikhistorikers beziehungsweise einer Mathematikhistorikerin müssen aber offenbar nicht um ein drittes, nämlich das Leben in den Theorien und Methoden der Geschichtswissenschaft und ihrer geistes- und sozialwissenschaftlichen Verwandten, ergänzt werden. Zumindest äußert sich van Brummelen zu dieser Problematik nicht. Dennoch sind Kontexte für den Autor wichtig. Er dokumentiert sie in seinem Buch durch Auszüge aus Originaltexten, die er doppelt, nämlich ins Englische und zudem in eine modernisierte mathematische Fachsprache übersetzt hat. Der Tücken solcher Adaptationen ist er sich wohl bewusst. Aber sein Verständnis dessen, was Kontext ist, erweist sich damit als sehr speziell. Es prägt die Themenwahl seines Buches ebenso wie die Sicht auf seine Vorgänger. Folglich richtet sich seine Aufmerksamkeit auf Personen, Methoden, Begriffe, Theoreme, Systematisierungen und Anwendungen, ohne jeden einzelnen Punkt in seinen fünf Kapiteln („Precursors“, „Alexandrian Greece“, „India“, „Islam“, „The West to 1550“) explizit aufzugreifen. Historiographische Themen wie Periodisierung, Interpretation, Kontextualisierung, Beziehungen zwischen verschiedenen Konzepten oder Positionen historischer Akteure hinsichtlich ihrer Konzepte, Methoden und Ziele greift er dagegen nicht wirklich auf.

\section{Neue Trends in der Wissenschaftsgeschichtsschreibung. Untersuchungen von Werken religiöser Gelehrter nach 1100}

Eine zentrale Schwäche der Forschung zur Wissenschaftsgeschichte in islamischen Gesellschaften besteht in dem fast ausschließlichen Interesse an 
Spitzenwerken, Innovationen und Texten aus den ersten drei bis vier Jahrhunderten. Als Resultat gibt es bisher nur sehr wenige systematische Untersuchungen zu mathematischen, astronomischen oder astrologischen Arbeiten religiöser Gelehrter, vor allem nach 1100, da diese sehr oft entweder Lehrtexte darstellen oder mathematische und astronomische Themen im Rahmen von religiösen Erörterungen behandeln. Umso erfreulicher ist es, dass Petra Schmidl, Robert Morrison und Amir Ljubović in ihren Büchern Beziehungen zwischen Religion und Wissenschaft und die Etablierung von Wissenschaft in neu eroberten Gebieten mit einer anderen Wissenskultur studieren.

Schmidl hat Texte von einem in Mekka im 11. Jahrhundert und zwei in Jemen im 13. Jahrhundert tätigen Gelehrten auszugsweise ediert und übersetzt, deren astronomische, meteorologische und religiöse Inhalte sie erläutert und kommentiert sowie ihre Beziehungen untereinander in Bezug auf zwei unterschiedliche astronomische Traditionen untersucht, die in islamischen Gesellschaften gepflegt worden sind (wissenschaftliche Astronomie, sogenannte volkstümliche Astronomie). Ihr ging es darum herauszufinden, was Autoren einer religiös konstituierten Diskussion von Himmelserscheinungen zu ihren Schriften veranlasst sowie zu ihrer Abfassung befähigt hat und wie sich deren Darlegungen, Arbeitsweisen und Ergebnisse von denen, die Kenntnisse und Erfahrungen in der wissenschaftlichen Tradition besaßen, unterscheiden. Darüber hinaus wollte sie ermitteln, ob diese Textsorte vielleicht Aufschlüsse über die Entstehung religiöser Praktiken und deren Sanktionierung in verschiedenen Regionen der islamischen Welt liefern kann. Während sie sich im Verlauf ihrer Arbeit vor allem mit einzelnen Wissensaspekten der ersten Frage befasst hat, ohne dabei die drei Autoren zusammenfassend zu vergleichen, schenkte sie religiösen Praktiken und ihren Beschreibungen in den drei Texten durchgängig Aufmerksamkeit.

Robert Morrison beschäftigt sich in seinem Buch mit dem Schaffen Nizām al-Dīn al-Nīsābūrīs ( $†$ ca. 1330), der sowohl als Religionsgelehrter als auch als Verfasser eines Werkes zur Planetentheorie bekannt geworden ist. Die Struktur seines Buches basiert auf dem methodischen Ansatz, die Wissenschaften den religiösen Disziplinen gegenüberzustellen und dann nach dem Einfluss der ersteren auf die letzteren zu fragen. Dennoch scheint Morrison zunächst aber davon auszugehen, dass der Konflikt zwischen den vorislamischen Wissensgebieten und den islamischen Disziplinen die Norm sei, während ihre Koexistenz oder gar gegenseitige Befruchtung die Abweichung von derselben darstelle. Seine Darstellung der Entwicklung der mathematischastronomischen Wissenschaften sowie der Astrologie leidet zum Beispiel unter dieser Sichtweise (S. 13-15). In den anschließenden Teilen seines Buches bemüht er sich aber vor allem um eine detaillierte Darstellung und Diskussion von Nīsābūrīs Ansichten zur Astronomie, Gottes Schöpfung und der Interpretation des Koran. 
Typisch für Morrisons Stil ist, dass er zwar von verallgemeinernden Positionen ausgeht, sie aber im Laufe seiner Detaildiskussionen oft abwandelt oder sogar ganz aufgibt. Ein wichtiges Beispiel dafür ist seine Schlussfolgerung, dass Nīsābūrīs Erfolg nicht von einer Zweiteilung zwischen seinem religiösen und wissenschaftlichen Werk abhängig gewesen sei. Wissenschaft sei ein Teil von Nīsābūrīs religiöser Gelehrsamkeit gewesen und die religiöse Gelehrsamkeit habe seine Wissenschaft konfiguriert (S. 146). Ein anderes, für die weitere Forschung zur Rolle der Astrologie und ihrer Beziehungen sowohl zur Astronomie als auch zum Spektrum der von Religionsgelehrten vertretenen Ansichten bedeutendes Ergebnis ist die Einsicht, dass Nīsābūrī der Astrologie eine Reihe von nützlichen, positiven Funktionen für die Kenntnis Gottes als auch für das Verständnis der theoretischen Astronomie zuerkannt hat (S. 68 f.). Morrisons Nachweis, dass Nīsābūrī aufgrund der Ähnlichkeiten, die er zwischen den demonstrativen Praktiken der Jurisprudenz und der Wissenschaften sah, zu anderen Positionen über den Status astronomischer Theorien gelangte als andere religiöse Gelehrte, die vornehmlich vom Kalām aus darüber reflektierten, trägt weitere Argumente zu der in der Philosophiegeschichte neu eröffneten Debatte zum Kausalitätsverständnis unter islamischen Religionsgelehrten bei (S. 70-77, 147). Seine Überlegungen zu Nīsābūrīs gleichzeitigen Bindungen an unterschiedliche religiöse Positionen (Schia, schafiitische Rechtsschule, ash'aritischer Kalam, Sufitum, sunnitische oder übergreifende Ideen) (S. 44-62) belegen den Wert von Detailstudien für das Verständnis lokaler Spezifika und gelebter Praktiken.

Ljubovićs Buch über Arbeiten zur Logik von bosnischen Autoren liefert einen Überblick über Handschriften von sieben bosnischen Gelehrten vom 16. bis ins 18. Jahrhundert, deren Inhalte, Methoden und Motivationen sowie ihre Beziehungen $\mathrm{zu}$ klassischen Traditionen der Logik in islamischen Gesellschaften. Insofern stellt es ein Novum in der Wissenschaftsgeschichtsschreibung dar. Auch wenn in seiner Darstellung vieles im Elementaren und Allgemeinen stecken bleibt, bieten Informationen und Vergleiche dennoch eine begrüßenswerte Erweiterung unserer Kenntnisse zu einer wichtigen Lehrdisziplin sowohl in islamischen als auch christlichen Gesellschaften. Das Buch kann somit als Grundlage für weitere Untersuchungen zur Ideengeschichte in verschiedenen Balkanstaaten in der frühen Neuzeit angesehen werden. Leider ist ein Teil der Handschriften, die Ljubović in seinem Buch besprochen hat, durch die Kriege in den 1990er Jahren vernichtet worden.

Ljubovićs Buch verdeutlicht außerdem in aller Schärfe ein generelles Problem heutiger akademischer Buchproduktion: das Fehlen eines Lektorats in den Verlagen. Fast alle hier besprochenen Bücher zeigen sprachliche und stilistische Schwächen, die im Vorfeld hätten korrigiert werden können. Die Übertragung der vollständigen Verantwortung für die Textproduktion an die Autoren und Autorinnen, inklusive der fremdsprachlichen Texte, hat $\mathrm{zu}$ einem unbestreitbaren Verlust an Qualität und Lesbarkeit geführt. 
Akademikerinnen und Akademiker sind eben nicht a priori auch gute Schriftstellerinnen und Schriftsteller, Übersetzerinnen und Übersetzer, Designerinnen und Designer, oder Computerfachleute. Im Falle von Ljubović ist das Englisch so schlecht ausgefallen, dass das Buch als Ganzes darunter leidet.

Die hier vorgestellten Bücher illustrieren die in der Einleitung beschriebenen Spannungen, denen die Wissenschaftsgeschichtsschreibung zu islamischen Gesellschaften seit wenigstens einem Jahrzehnt ausgesetzt ist. Sie markieren die Aspekte und Arbeitsweisen, die sie momentan sowohl von der Philosophiegeschichtsschreibung in diesen Gesellschaften als auch von der Wissenschaftsgeschichtsschreibung für andere Kulturen und Perioden trennen. Die Arbeiten jüngerer Kolleginnen und Kollegen zeigen erfreulicherweise, dass diese Probleme inzwischen produktiv reflektiert werden und die Rezeption neuerer Ansätze eingesetzt hat.

\section{Sonja Brentjes}

Departo. de Filosofía y Lógica

Universidad de Sevilla

c/Camilo José Cela s/n

Seville 41018

Spain

E-Mail: brentjes@us.es 